Published in final edited form as:

Curr Opin Immunol. 2013 April ; 25(2): 189-191. doi:10.1016/j.coi.2013.02.012.

\title{
Tumor Immunology
}

\author{
Glenn Dranoff ${ }^{1}$ and Douglas Fearon ${ }^{2}$ \\ Glenn Dranoff: glenn_dranoff@dfci.harvard.edu; Douglas Fearon: dtf1000@cam.ac.uk \\ ${ }^{1}$ Department of Medical Oncology and Cancer Vaccine Center, Dana-Farber Cancer Institute and \\ Department of Medicine, Brigham and Women's Hospital and Harvard Medical School, Boston, \\ MA 02215 \\ ${ }^{2}$ CRUK Cambridge Research Institute, Li Ka Shing Centre, Robinson Way, Cambridge CB2 ORE, \\ UK
}

Cancer immunology has matured into a vibrant discipline, impacting both basic cancer research and clinical oncology. Investigations into the host-tumor relationship have delineated a complex interplay of cancer cells, stromal elements, and immune components. Within the tumor microenvironment, a broad spectrum of host responses may be generated, which may range from protective cytotoxic reactions to tumor-promoting inflammatory reactions. The specific factors that dictate the outcome of the anti-cancer response remain under active study, but tumor cells typically exploit host factors to foster disease progression and create a local immunosuppressive network. The elucidation of some of the critical pathways restraining protective immunity has given rise to novel immunotherapies that target specific defects in the host reaction. The clinical successes of immune checkpoint blockade (with blocking antibodies to the negative T cell costimulatory molecules CTLA-4 and PD-1), cancer vaccines (with dendritic cells pulsed with tumor antigens), and T cells engineered with chimeric antigen receptors (anti-CD19 for lymphoid leukemias) validate a key role for immunity in tumor pathogenesis. This issue of Current Opinion in Immunology brings together leading investigators to review rapidly developing areas in cancer immunology and immunotherapy.

The two contributions by DuPage and Jacks, and Vonderheide and Bayne emphasize the importance of using genetically engineered mouse models (GEMs) to study the host immune response to cancers. Although these models cannot precisely replicate all aspects of human cancers, they do present many of the genetic and histological features of the human cancers that they have been designed to reproduce. In some instances, the GEM models even demonstrate the characteristic pre-malignant phases of these diseases, and the occurrence of early metastatic lesions. The GEM models also replicate the non-cancer cell, stromal components of the tumors, which is highly relevant in that the immune system is a major stromal element.

DuPage and Jacks discuss the importance of the type of antigens and the timing of their expression by GEM models, with an ultimate goal being the inducible expression of a tumor-specific or tumor-associated antigen after the development of the tumor microenvironment. Their contribution also points out that the tissue context of the tumor may have a decisive role in the means by which the tumor evades the host immune response. Their experiments have shown, for example, that during the development of a sarcoma, the phenomenon of cancer immunoediting dominates the interaction between the cancer and the host immune response, whereas an epithelial tumor having the same cancer-inducing mutations escape immunosurveillance by a process involving the impaired function of effector T cells. Finally, these authors discuss how the use of GEM models permits an analysis of the effects of combining immunotherapy with conventional cancer therapies. 
Vonderheide and Bayne review their findings demonstrating that the so-called "KPC" GEM model for pancreatic ductal adenocarcinoma has relevance to an understanding of the role of innate and adaptive immunity in the human form of the disease, and also in other cancers. For example, they point out that in this spontaneous cancer model, $\mathrm{T}$ cells are rarely observed in the cancer lesions whilst there is marked infiltration of myelomonocytic and granulocytic cells. This characteristic of limited T cell localization to cancer cells nests has been reported for several other human cancers, including ovarian and colorectal. This GEM model has also permitted this research group to show that these innate immune cells can both protect the tumor from adaptive immune control, and, paradoxically, mediate control of tumor growth without the assistance of the adaptive immune system after appropriate stimulation. Thus, their work reinforces the theme that the immune system has two potential roles in tumors, a circumstance that may reflect its interactions with injured and infected normal tissues, respectively; that is, repair in the former circumstance, and elimination of immunogenic cells in the latter.

Charles Dimitroff discusses the role of galectin-1 in tumor immune escape. A variety of cancers secrete high levels of galectin-1, which binds to $\mathrm{N}$-acetyllactosaminyl glycans expressed on the surface of $\mathrm{CD}^{+} \mathrm{Th} 1$ and $\mathrm{CD} 8^{+}$cytotoxic $\mathrm{T}$ cells. This engagement promotes apoptosis and/or inhibition of effector function, whereas FoxP3 ${ }^{+}$Tregs that lack these glycans are spared. Fluorinated glucosamine analogs were initially developed as direct anti-tumor cytotoxic therapies, but recent work indicates that they may inhibit the production of $\mathrm{N}$-acetyllactosamines on $\mathrm{T}$ cells, perhaps through altering the intracellular pools of uridine diphosphate $N$-acetylglucosamine. The systemic administration of fluorinated glucosamine analogs promotes tumor destruction through augmenting cytotoxic T cell activity. Nonetheless, because the impact of these compounds on the function of antigen presenting cells and hematopoietic cells remains to be clarified, an exciting therapeutic application involves the treatment of $\mathrm{T}$ cells ex vivo prior to adoptive transfer. This approach might also attenuate $\mathrm{T}$ cell suppression mediated through galectin-3 and -9 , which are also frequently expressed in the tumor microenvironment.

Weiping Zou and colleagues highlight the dynamics of $\mathrm{T}$ cell functional states in cancer, focusing on anergy, exhaustion, senescence, and stemness. Anergy is characterized by deficient IL-2 production and a limited replicative capacity, which may be triggered through $\mathrm{T}$ cell receptor engagement in the absence of positive costimulation and/or an excess of negative costimulation. Anergy involves the negative regulation of early $\mathrm{T}$ cell signaling and epigenetic modifications that inhibit key transcriptional effector programs. Exhaustion arises under conditions of chronic stimulation, resulting in impaired cytokine production and cytotoxicity in response to specific antigen. Inhibitory B7 family members, particularly PD-1, and other surface proteins including Tim-3, LAG-3, 2B4, CD160, and BTLA contribute to this state. Senescence may be elicited by telomere shortening, leading to cell cycle arrest, limited effector function, and elevated levels of negative regulators. Stemness reflects a dual capacity for self-renewal and proliferation/differentiation into long-lived effector cells. T cell surface proteins that regulate these various functional states are attractive targets for antibody-based immunotherapies.

Carl Ware and colleagues illuminate the roles of lymphotoxin- $\alpha \beta$, LIGHT, and their receptors in tumor immunity. This network of TNF and Ig superfamily members participates in communication between lymphoid cells and stroma elements, which is essential for effective innate and adaptive immunity. Persistent activation of this network may underlie some forms of tumor-promoting inflammation, as chronic lymphotoxin expression drives hepatocellular carcinoma formation and prostate carcinoma progression in mice. Mutations in some family members such as HVEM, BTLA, and CD160 are found in hematologic and epithelial malignancies, underscoring the importance of this network in oncogenesis. 
However, the specific roles of HVEM in tumor formation may be complex. While HVEM may attenuate $\mathrm{T}$ cell immunity and promote tumor cell survival in melanoma, loss-offunction mutations are frequent in B cell lymphomas, where they may result in unopposed lymphotoxin signaling and persistent inflammation.

Andrew Weinberg and colleagues focus on therapeutic strategies targeting OX40, 4-1BB, and CD40, which are members of the TNFR superfamily expressed on T cells and/or antigen presenting cells. Engagement of these receptors by specific ligands or agonistic monoclonal antibodies augments $\mathrm{T}$ cell proliferation, activation, survival, and effector function. Signaling through these TNFRs proceeds through TRAFs that engage downstream NF- $\kappa \mathrm{B}$, MAP kinase, and PI3-kinase pathways. In murine models, agonistic antibodies to OX40 promote tumor destruction through enhanced $\mathrm{CD} 4^{+}$and $\mathrm{CD} 8^{+} \mathrm{T}$ effector function, although the impact of Tregs is still under active study; Phase I clinical testing of antibodies to human OX40 has been initiated. Agonistic antibodies to 4-1BB similarly promote long-lived antitumor $\mathrm{CD}^{+}$cytotoxic $\mathrm{T}$ cell responses in murine models and may additionally activate endothelial cells to increase leukocyte trafficking. Clinical studies with antibodies to human 4-1BB are also underway, with early results establishing both anti-tumor activity and a potential for serious toxicity. CD40 may be targeted with either agonistic antibodies, which can promote immune mediated destruction of melanomas and pancreatic carcinomas in patients, or antagonistic antibodies that block CD40 mediated survival signaling in tumor cells and promote antibody-dependent cell cytotoxicity or phagocytosis by innate cells.

Weiwei Li and David Mooney discuss novel materials-based strategies for cancer immunotherapy. Biomaterial platforms enable precise special and temporal control over the release of immunomodulators, resulting in sustained host responses while reducing systemic toxicities. Three dimensional, porous scaffolds may be multi-functional through incorporation of several components; for example, the inclusion of GM-CSF and CpGs together promote efficient dendritic cell activation. Micro- and nanoparticles may be fabricated to act as vaccines that target cancer antigens to particular cells and subcellular organelles. The application of $\mathrm{pH}$ and oxidation-reduction chemistry to these particles directs the release of immune payloads specifically in endosomes. Nanoparticles may also be directly conjugated to the surface of T cells prior to adoptive therapy to augment cell function through the autocrine/paracrine delivery of cytokines or small molecules that inhibit negative regulatory pathways.

Dean Felsher and colleagues discuss their intriguing finding that oncogene addiction is not strictly cell autonomous, but also involves a major role for host immunity. The acute interruption of a cancer cell driver mutation in vivo remodels the tumor microenvironment and stimulates innate and adaptive immunity that are critical to sustained tumor destruction. In Myc and Bcr-Abl driven murine tumor models, loss of oncogene function elicits a CD4 ${ }^{+}$ $\mathrm{T}$ cell response that modulates the induction of tumor cell senescence and inhibits angiogenesis; thrombospondin-1 is one critical mediator of these actions. The principle of crafting therapies that target tumor cell intrinsic defects and host responses is also illustrated through the detailed analysis of clinical response to c-Kit inhibitors in patients with gastrointestinal sarcomas and Braf inhibitors in patients with advanced melanoma; loss of oncogenic function is coupled to immune activation, resulting in more potent anti-tumor effects.

Taken together, the eight reviews in this section of Current Opinion highlight the substantive progress in cancer immunology and immunotherapy. This deeper understanding of host-tumor interactions provides a richer framework for devising efficacious treatments that may improve clinical outcomes. 


\section{Biographies}

Glenn Dranoff

Department of Medical Oncology and Cancer Vaccine Center, Dana-Farber Cancer Institute and Department of Medicine, Brigham and Women's Hospital and Harvard Medical School, Boston, MA 02215. e-mail: glenn_dranoff@dfci.harvard.edu

Glenn Dranoff is Professor of Medicine at Dana-Farber Cancer Institute and Harvard Medical School and Leader of the Dana-Farber/Harvard Cancer Center Program in Cancer Immunology. His laboratory focuses on elucidating the cellular and molecular mechanisms underlying the generation of anti-tumor immunity.

Douglas Fearon

CRUK Cambridge Research Institute, Li Ka Shing Centre, Robinson Way, Cambridge, CB2 0RE UK, e-mail: dtf1000@ @am.ac.uk

Douglas Fearon is Emeritus Sheila Joan Smith Professor of Immunology and Senior Group Leader of the Cambridge Research Institute at the University of Cambridge. His research interests have included innate immunity, B cell signaling, the development of memory $\mathrm{T}$ cells, and, most recently, the role of the stromal microenvironment in tumoral immune suppression. 